\title{
'An investigation of the nervous control of defecation' by Denny- Brown and Robertson: a classic paper revisited
}

\section{J. A. Vilensky*, D. R. Bell† and S. Gilman}

Departments of *Anatomy and Cell Biology and †Physiology, Indiana University School of Medicine, Fort Wayne and łDepartment of Neurology, University of Michigan School of Medicine, Ann Arbor, USA

Received 25 September 2003; accepted 25 September 2003

\begin{abstract}
In 1935 two young neurologists, Derek Denny-Brown and E. Graeme Robertson, published an article explaining the mechanisms underlying human defaecation based on a manometric study in patients with sacral root and spinal cord lesions, and normal subjects. This article is still routinely cited in studies of rectal and sphincter ani function. Unfortunately, however, the article itself is not written well, being composed of long convoluted sen-

tences and containing 79 often indecipherable figures. Difficult-to-understand articles were common to the publications of Denny-Brown, who became one of the most prominent neurologists of the twentieth century. In accord with our prior work explaining Denny-Brown and Robertson's earlier paper on micturition, we provide here what we hope is a clear explanation of the methods and results in their study on defaecation.
\end{abstract}

\section{Introduction}

Derek Denny-Brown (1901-81; Fig. 1) was one of the renowned neurologists of the twentieth century [1]. Shortly after completing his doctoral dissertation at Oxford University under a prominent neurophysiologist, Sir Charles Sherrington, he and fellow neurologist E. Graeme Robertson (1903-75; Fig. 1) initiated clinical studies designed to understand the neurophysiology of micturition and defaecation [2,3]. Although well-cited even to the present day, both of these studies are difficult to read and comprehend because of poor organization, inconsistencies in terminology, frequent repetition, excessive extraneous information, convoluted sentences and many, very difficult to decipher, illustrations (which are mainly photographs of the light-sensitive paper used to record the manometer values). For example, the defaecation article is 50 pages long and contains 79 figures, which are cited out of order.

As part of our effort to elucidate Denny-Brown's articles to a larger and contemporary audience, we have previously published articles pertaining to his views on the underlying basis of certain neurological diseases [4-6] and have recently published an easier-to-understand

Correspondence to: Dr Joel A. Vilensky, Indiana University School of Medicine, 2101 Coliseum Blvd. E., Fort Wayne, IN 46805, USA.

E-mail: vilensk@ipfw.edu version of his micturtion article [7]. Here we attempt a similar explanation of his defaecation article.

At the time Denny-Brown and Robertson began their defaecation research, little information was available about the role of local as compared with central processes in the control of defaecation. Thus, they were particularly interested in understanding the mechanism by which the sphincter ani relaxes, permitting defaecation to occur. Moreover, little was known about the differences in the abilities and control of smooth muscle as compared with striated muscle. The ultimate aim of the research was to develop better means of treating and managing patients with neurological deficits in the control of defaecation.

In this review we describe the remarkable methodology the investigators developed to study defaecation, and explain their major findings by reviewing and explaining each of their 14 conclusions. We have redrawn some of the figures presented in the paper including elaborating the method illustrations. For the redrawn data figures, we extrapolated the values from the graphs presented; thus, the curves are similar, but not identical to the originals. The legends to the figures indicate the number of the original figure from which each of our figures was derived.

\section{Methods}

Denny-Brown and Robertson investigated three groups of subjects. The first two groups consisted of patients 

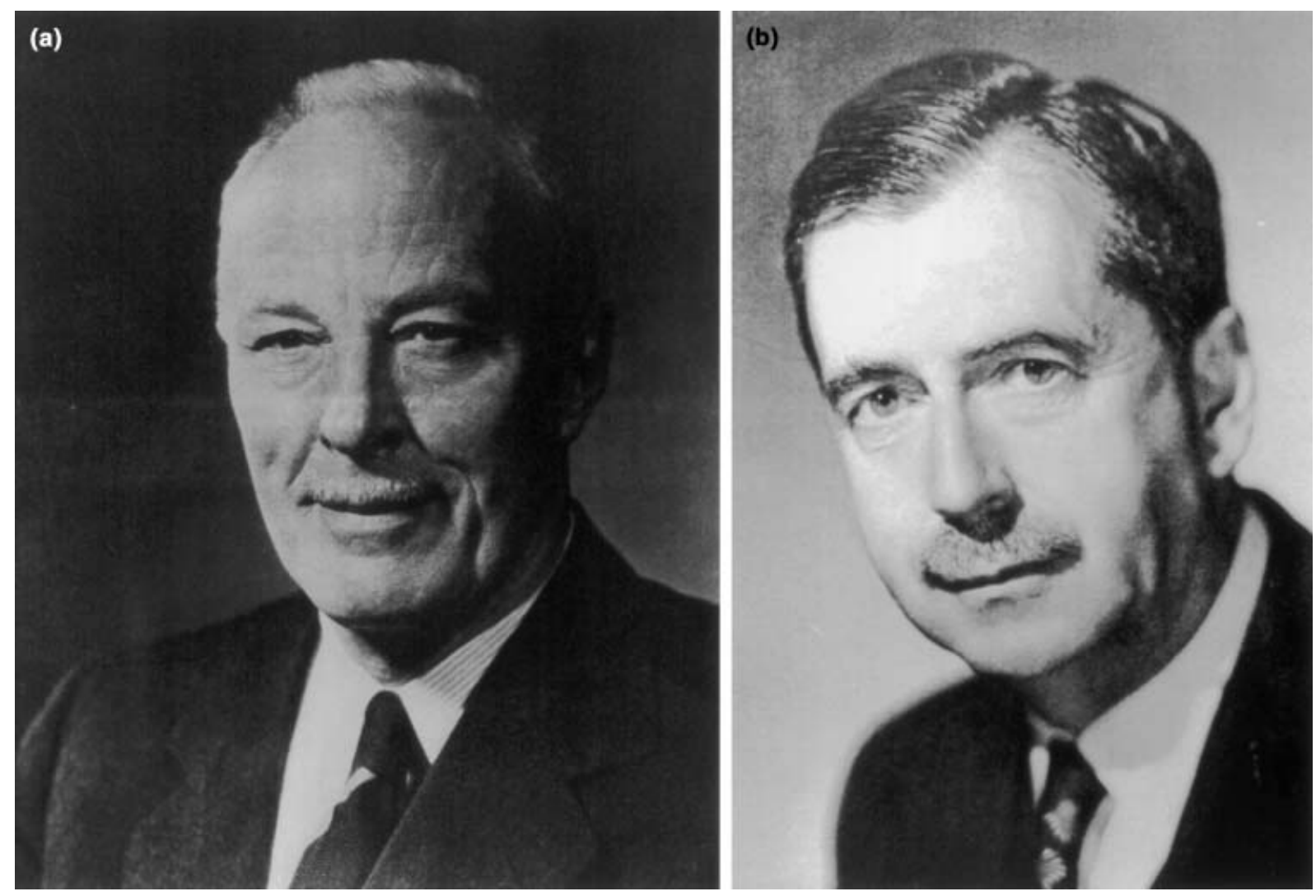

Figure I Photographs of Drs Denny-Brown (a) and Robertson (b).

with difficulty in defaecation associated with lesions of the sacral nerve roots and of the spinal cord, whereas the third group consisted of two normal individuals. As with the earlier micturition study, the two investigators served as the normal subjects.

The authors did not describe the two patient groups in the 'Methods' section, but rather under the headings, 'The effects of lesions of the sacral roots', and 'Transverse lesions resulting in reflexly active states. The investigators distinguished the two groups based upon postsurgical reports and the results of neurological examinations. Both groups had heterogenous disorders. Group 1 consisted of four patients with sacral nerve root lesions or similar kinds of pathology whereas Group 2 consisted of seven patients with spinal cord injury. In addition, in the 'Discussion' section of the article (which was not titled as such) two additional patients were discussed: a patient with recent spinal cord injury who was in spinal shock and a patient with megacolon (now known as Hirschsprung's disease or aganglionic megacolon) who had undergone section of his hypogastric (presacral) nerves.

The investigators studied defaecation processes in most, but not all subjects, by inserting a rubber balloon into the rectum, a specially designed balloon into the anus and a catheter for passing saline into the space intervening between the two balloons. A third balloon was secured to the abdomen to measure abdominal movements.

The simple rubber balloon inserted into the rectum could be inflated through a catheter that was attached to a syringe (Figs 2 and 3 ). This device was used to measure rectal wall pressure, which reflected muscle force. The distal end of the catheter was attached to a mirror manometer that moved in direct response to the pressure exerted on the balloon. The movements of the mirror were recorded on light-sensitive recording paper, and the movements could be calibrated (Fig. 2).

The device inserted into the anus contained inflatable rubber membranes that were attached to a brass 'obturator' (Fig. 3). The authors used this sophisticated device to measure accurately the response of the involuntary sphincter to stretch. Similar to the rectal balloon, the anal (sphincter) balloon (obturator) was attached to a catheter that could inflate it and also measure the force exerted by the involuntary sphincter.

The third measuring device consisted of a catheter with an open end placed in the 'intervening' space 


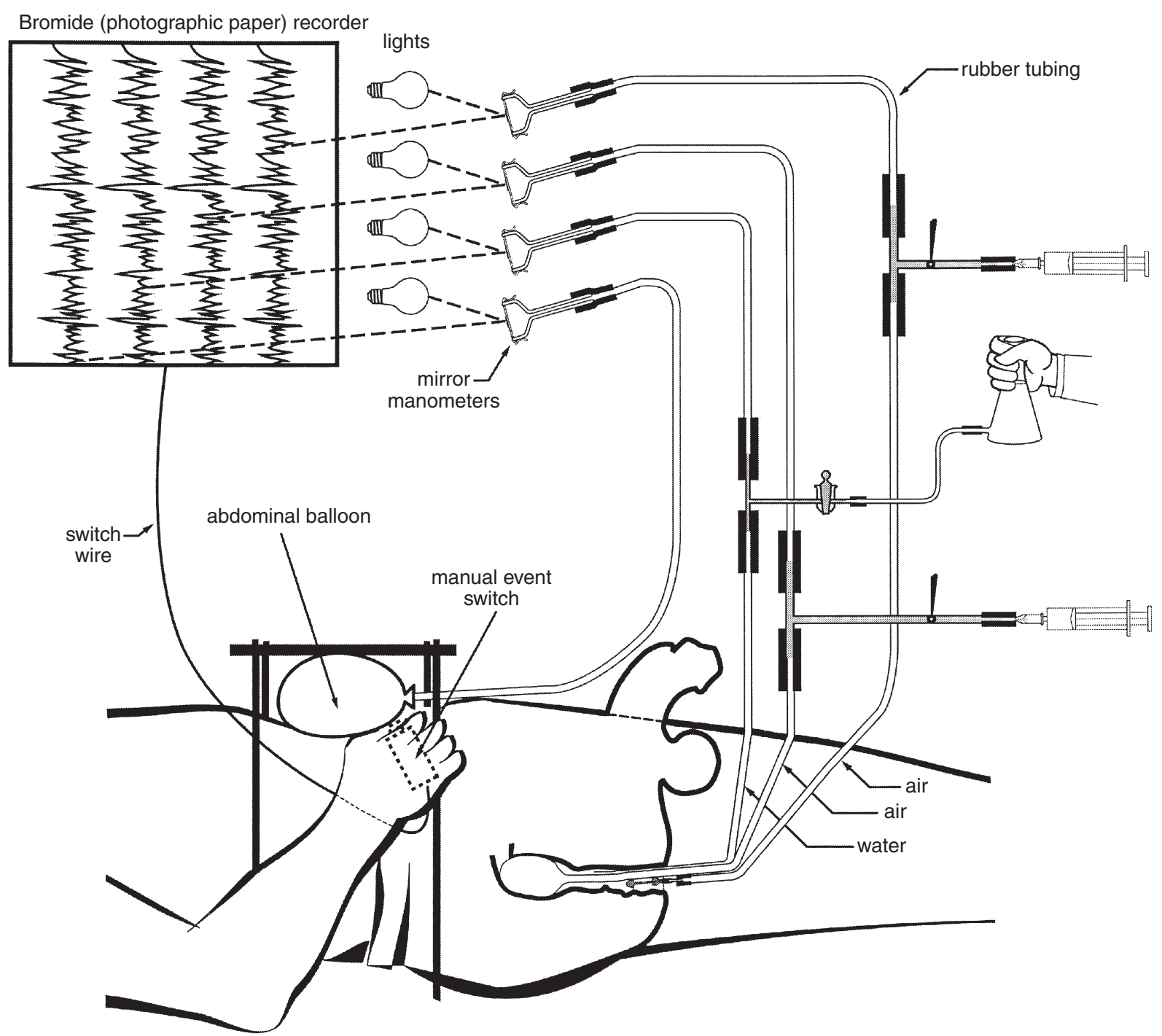

Figure 2 Depiction of the typical experimental set-up (cf. text for details and Figure 3 for close-up of the rectal/anal sensors).

(between the rectal and anal balloons). The investigators allowed saline to flow into this space through the catheter, which, similar to the other catheters, also served as a pressure measuring device (Fig. 2). Introduction of fluid through this catheter allowed fluid pressure to be applied to a large area of the rectal canal. Lastly, abdominal movements were measured by a balloon/catheter attached to the abdominal wall (Fig. 2).

At times while the investigators were taking measurements, they asked the subjects to try to defaecate, which occurred on some occasions. This was to determine the effects of voluntary efforts to defaecate on the reactions studied. In both the present account and the original report, the term, 'sphincter ani' refers only to the 'smooth' or involuntary part of the muscle and the term, 'voluntary' sphincter refers to the striated part of the muscle. The paper contains two definitions that are important for understanding the conclusions of the study, but that are somewhat buried within the original text. 'Automatic' defaecation refers to the process that occurs in individuals in which all neuronal input from the spinal cord to the rectum and anus has been destroyed. 'Reflex' defaecation occurs in spinal-cord injured individuals who have functioning rectal/anal neuronal circuits.

\section{The conclusions}

For the 14 'Conclusions' presented below, we paraphrase and underline the description of each provided in the original article (pp. 305-6), and then usually provide 
a

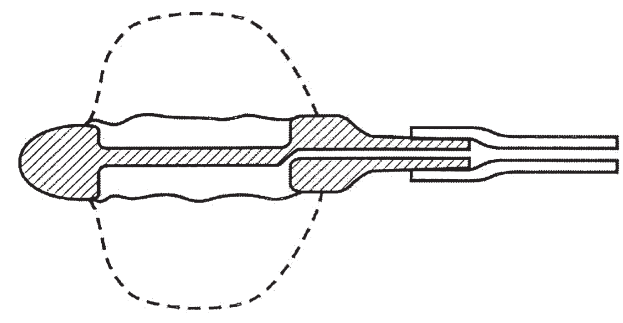

Figure 3 (a) Enlarged illustration of the balloon-like device that the authors developed to distend and measure pressure within the sphincter ani; the dashed line shows the 'balloon' in an inflated configuration. (b) Enlarged view of the catheters and sensors in the rectum and anal canal (modified from original Figure 1).

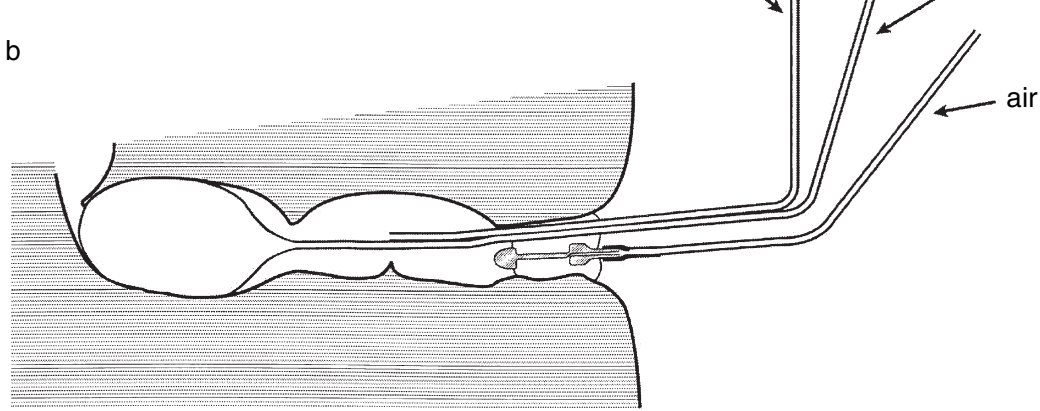

additional explanations (sometimes with accompanying figures) to better explain the points the authors were trying to convey.

\section{In automatic defaecation there is a reciprocal relationship between contraction of the rectum and relaxation of the anal sphincter}

Denny-Brown and Robertson along with Gowers (1877) [8] defined the 'rectal-anal inhibitory reflex' (RAIR; Fig. 4). Initially, the investigators inflated the anal (sphincteric) balloon with $5 \mathrm{cc}$ of air to distend the

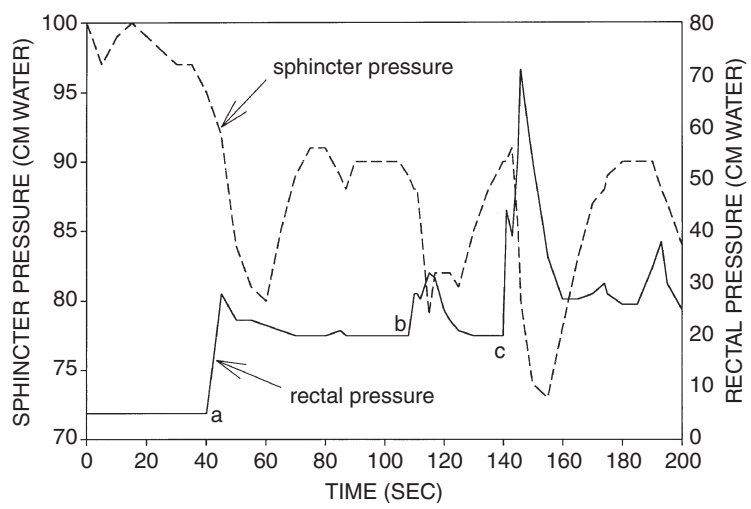

Figure 4 From case 1 who was diagnosed with compression of the cauda equina by a neurofibroma. The rectal balloon was suddenly inflated with $5 \mathrm{cc}$ air at a, and further inflated with 5 and then $10 \mathrm{cc}$ at b and c, respectively. Note slightly delayed decreases in sphincter pressure (-.--) with each rectal inflation $(-)$ (modified from original Fig. 26). sphincteric muscle moderately. At time ' $a$ ' in the figure, they suddenly inflated the rectal balloon with $5 \mathrm{cc}$ of air, with a further $5 \mathrm{cc}$ at $\mathrm{b}$ and $10 \mathrm{cc}$ at $\mathrm{c}$. With each inflation of the rectal balloon the sphincter relaxed, as shown by the reduction in pressure. Further, the relaxation was slightly delayed, suggesting to Denny-Brown and Robertson that the relaxation was associated with the onset of a rectal contraction wave.

2. Neural connections account for the reciprocal relationship between the rectum and involuntary sphincter. The required stimulus is tension upon the walls of the rectum. Active tension is more powerful than passive tension

Denny-Brown and Robertson's use of the term 'active tension' suggests that they believed rectal contraction is required to initiate a strong sphincter relaxation. This is not consistent with current views in which it is the passive stretch of the rectal wall that is believed to initiate a sequence of rectal contraction followed by sphincter relaxation [9].

3. The nervous mechanism for this 'reflex' reaction (i.e. the RAIR) appears to be related solely to intrinsic neural connections within the walls of the rectum and anal canal

The appearance of this relationship in the patients with cauda equina lesions demonstrated that the RAIR is not dependent on spinal or cerebral input. 


\section{The RAIR becomes depressed for a brief period during spinal shock when the sphincter ani relaxes only slightly in response to rectal distension}

This conclusion was based on case 12 , a 60 year-oldman who became paraplegic from a fall, with loss of all sensation and movement below the waist. He was studied 4 days after the injury and died 7 days after that. This patient was examined at bedside, consequently some of the techniques differed from those utilized in the laboratory. Figure 5 contains data from this individual, showing only slight relaxation of the sphincter after injection of $10 \mathrm{cc}$ of air into the rectal balloon, which had previously been inflated with $50 \mathrm{cc}$ of air. This patient's sphincter showed no reaction to inflation below $50 \mathrm{cc}$ and the rectum did not exhibit any contractile activity or passive relaxation following a sudden distension. Thus, compared to the patient represented in Fig. 4 the rectum and sphincter in the spinal shock patient are much less responsive to stimulation.

\section{Reflex defaecation results in a more complete rectal response to tension than automatic defaecation}

The maximum pressure from rectal contraction reached $40-50 \mathrm{~cm}$ of water in automatic defaecation, whereas in reflex defaecation pressures reached $60-100 \mathrm{~cm}$. In normal individuals, the pressures ranged between 100 and $200 \mathrm{~cm}$. The authors commented that the pressures during automatic defaecation would be sufficient only to expel fluid.

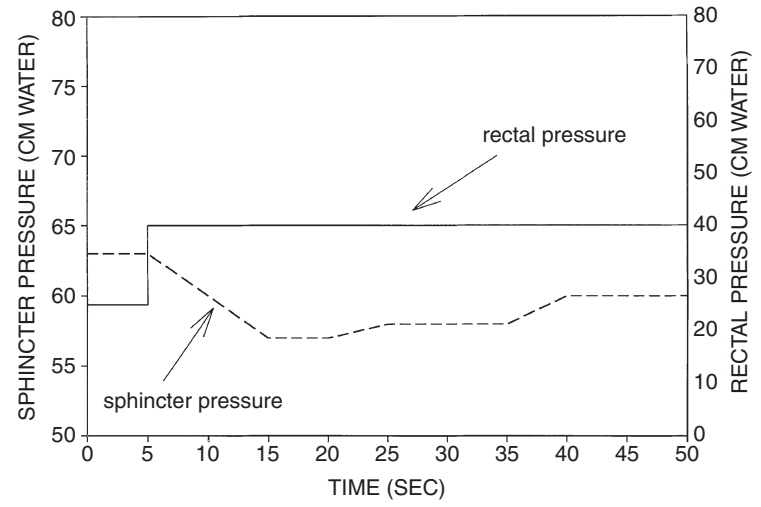

Figure 5 From case 12 who had a spinal dislocation at Tl0 and was in spinal shock at the time of the examination. Note the relatively slight decrease in sphincter pressure (----) in response to the rapid increase in rectal pressure $(-)$ at $5 \mathrm{~s}$ resulting from an injection of $10 \mathrm{cc}$ of air into the rectal balloon (pressure ranges are purposely the same as in Figure 4 so that differences can be noted; cf. text; modified from original Fig. 79).

\section{Reflex defaecation can be facilitated by cutaneous stimulation in the skin area of the sacral segments, with maximal effect at the anus, and activity of the bladder}

This is self-explanatory.

7. The sympathetic nerve supply from the hypogastric nerves to the rectum and anus does not participate in the control of defaecation

This conclusion is based on case 13, who had surgical resection of the hypogastric nerves after a diagnosis of megacolon. Following surgery, the patient's rectum and sphincter reacted in typical fashion except for differences in values that were related to the increased rectal capacity. Thus, Denny-Brown and Robertson concluded that the rectum, similar to the rest of the bowel, functions independently of the sympathetic nervous system.

8. The 'postural' tone of the rectum and sphincter ani is a reaction to passive tension of the muscle concerned. In each, a rapidly increasing tension causes tonic contraction, which then becomes phasic so that tension also becomes the stimulus for phasic contraction

Following sudden distension, the sphincter ani reacts with a sharp initial increase in pressure followed by rapid relaxation to a low pressure for 10-20 s, finally followed by a gradual rise to a higher level (Fig. 6). The latter rise shows some fluctuations in pressure that gradually fade, leaving a residual steadily maintained pressure, which is the postural tone of the muscle. The authors related these

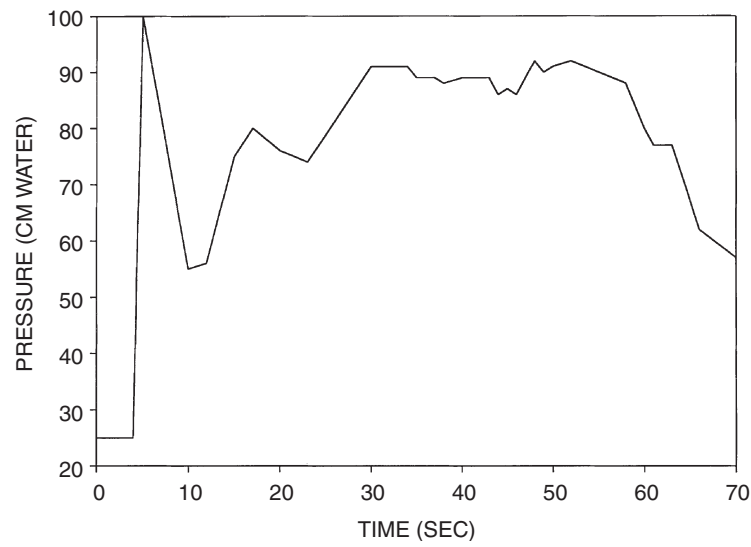

Figure 6 From case 2 who had arachnoiditis involving the cauda equina. The pressure changes in the sphincter ani are shown in response to sudden distension by injection of $20 \mathrm{cc}$ of air at approximately $5 \mathrm{~s}$ into the balloon located at the sphincter (cf. text; modified from original Figure 3 ). 
changes to a momentary resistance of the involuntary muscle to sudden stretch, followed by maintained contraction of the muscle upon the contents of the lumen. The authors attributed some small part of this reaction to the visco-elastic nature of the tissues concerned, but the majority to active muscle contraction, which is consistent with contemporary understanding of smooth muscle function.

Denny-Brown and Robertson also examined the reaction of the sphincter to incremental increases in pressure of lcc (Fig. 7). They showed that the sphincter force increases with each lcc distension of the balloon, introduced at 8 second intervals. After a certain degree of distension, however, the sphincter does not react with further increases in pressure. Thus, after the introduction of between 5 and $20 \mathrm{cc}$ of air the pressure exerted by the sphincter was largely 'plastic' and 'adaptive', remaining at about $80 \mathrm{~cm}$ (before micturition). The level of adaptation varied between patients and even within the same patient. Thus the 'after micturition' curve in the figure represents the same patient after micturition had occurred, showing adaptation at about $60 \mathrm{~cm}$ to the distension caused by the injection of air into the sphincter balloon. Similarly, Fig. 8 shows that the sphincter ani was less resistant to stretch in normal individuals when the desire to defaecate was present. Denny-Brown and Robertson related the low resistance and high degree of adaptation of the sphincter to the 'soft, patulous feel' during digital examination.

\section{The tone of the sphincter ani depends only on} intrinsic neural connections within the distal colon and variation reflects the degree of activity of the rectum

The similarity in responses among the patients demonstrated this to be the case, and Fig. 7 illustrates how

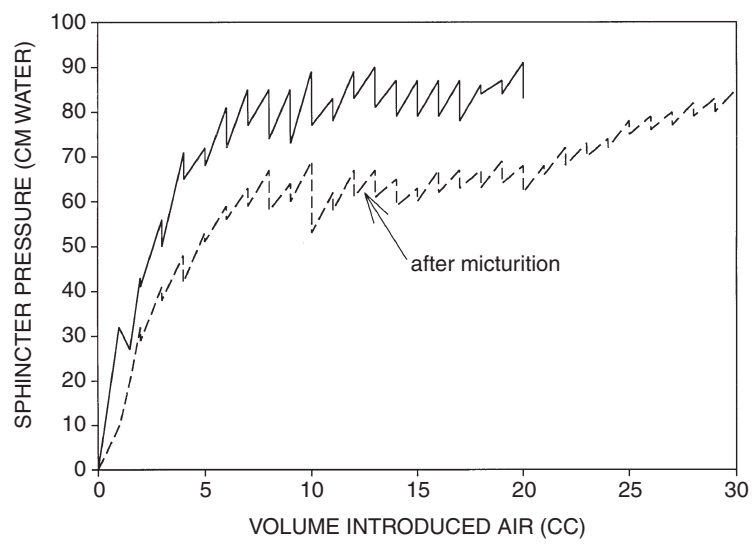

Figure 7 From case 2 who had arachnoiditis involving the cauda equina. The variability in sphincter pressure in response to sphincter distension in lcc intervals, every $8 \mathrm{~s}$, before $(-)$ and after (----) micturition (cf. text; modified from original Fig. 13).

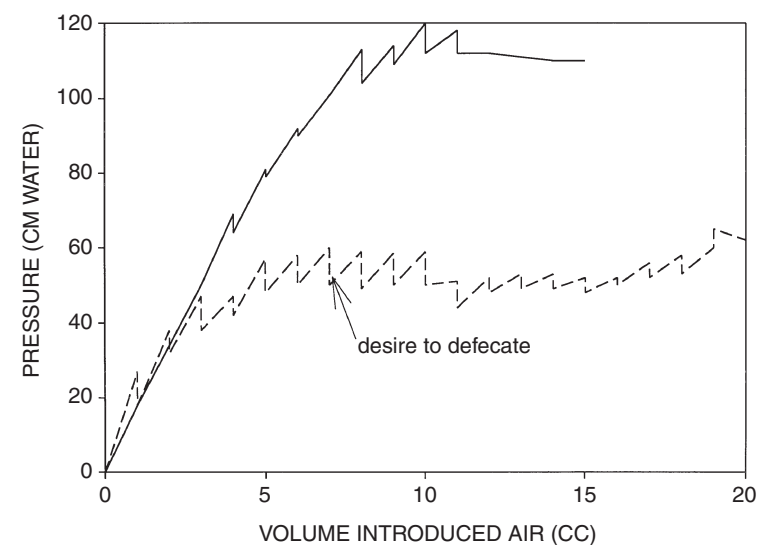

Figure 8 From one of the normal subjects. This demonstrates the reduction in sphincter reaction to distension that occurs with a desire for defaecation (modified from original Fig. 22).

changes in excitability of the sphincter (in this case associated with micturition) can change the level of adaptation in this muscle.

\section{Voluntary control over defaecation extends directly only to the external voluntary sphincter}

The authors found no evidence of activity of the rapidly contracting voluntary sphincter in the patients with cauda equina lesions, some evidence of activity in the patients with spinal cord injuries, and abrupt contraction of this muscle in the normal subjects when they tried to constrain evacuation.

\section{The voluntary sphincter is not tonic (in sustained contraction), although it contracts reflexly with the flexion reflex, and in synergy with the abdominal wall}

The flexion reflex occurs as a response to nociceptive stimulation in paraplegic people and consists of involuntary marked flexion of the lower extremities coupled with abdominal contraction. Whereas the external sphincter and levator ani are momentarily activated with the flexion reflex (the anal reflex), the internal sphincter is not activated. Reflex flexion in response to noxious stimuli was found to facilitate defaecation indirectly via abdominal contraction (cf. below), which sufficiently increased rectal tension.

12. Defaecation depends primarily on the reaction of the rectum to distension

This is self-explanatory. 
13. Weak rectal contractions explain the inadequacy of defaecation in spinal-cord-injured patients

This is self-explanatory.

14. The processes involved in defaecation and micturition are very similar. One difference, however, is that whereas contractions of the bladder can be inhibited voluntarily, rectal contractions are not subject to any voluntary influence

This is self-explanatory [7].

\section{Discussion}

Two major findings from this investigation were not integrated into Denny-Brown and Robertson's 14 Conclusions. The first involved the introduction of fluids into the 'intervening space.' Introduction of fluid into a patient with compression of the cauda equina by a neurofibroma resulted in a series of waves of rectal contractions that decreased in intensity when the flow was stopped. The sphincter ani maintained a relaxed posture during the inflow period. The pressure wave within the intervening segment occurred simultaneously with that of the rectum, indicating that the whole rectal canal contracts simultaneously and not in a peristalic wave. Further, the introduction of fluid induced a large rectal contraction. This suggested to the authors that, because the fluid stretched a larger area of the rectum than the balloon, it resulted in greater rectal distension and thereby more intense sphincter relaxation. In this patient, the reciprocal contraction of the rectum and relaxation of the sphincter resulted in evacuation of the fluid.

The second finding involved movements of the abdominal wall. Denny-Brown and Robertson did not elaborate on this aspect of the project, but did report that in one of the patients with spinal cord injury, abdominal pressure did induce rectal contraction and sphincter relaxation (Fig. 9). Also, in the normal subjects, voluntary efforts to defaecate occurred via abdominal contractions that in turn stimulated rectal contractions. Thus, even with a filled rectum, when one of the normal subjects tried to defaecate without abdominal contraction, he could not produce evacuation.

The most important and frequently cited finding of this paper is the RAIR, which Gowers had described in 1877 [8]. The contemporary view is that the temporary relaxation of the internal sphincter in response to rectal distension constitutes an important part of the continence mechanism [10].

Even though almost 80 years have elapsed since the publication of Denny-Brown and Robertson's report,

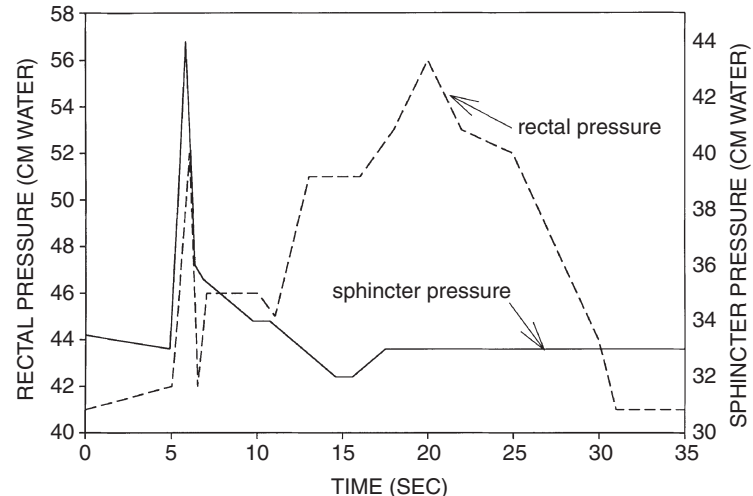

Figure 9 From case 7 who had complete destruction of L2-5 segments, and partial lesions at T11-Ll. At $5 \mathrm{~s}$, a brief 'passive' pressure was applied to the abdominal wall. This results in the subsequent increase in rectal pressure (-.--) and decrease in sphincter pressure (-) (modified from original Fig. 48).

controversy remains pertaining to the pathways involved in mediation of the RAIR. Denny-Brown and Robertson suggested that the reflex was mediated via intramural connections. The absence of this reflex in Hirschsprung's disease, which is characterized by the absence of ganglion cells in segments of the colon and rectum, supports their findings [11]. Further, recent studies have also confirmed the finding that the reflex persists after section of the hypogastric nerves [12]. Nevertheless, another study demonstrated a $50 \%$ decrease in anal pressure after blockade of sympathetic input, and in vitro studies have identified excitatory alpha-adrenergic receptors, inhibitory beta-adrenergic receptors, inhibitory or biphasic cholinergic receptors and inhibitory noncholinergic, nonadrenergic receptors located in sphincter. Additional experimental evidence provides support for both alpha (constrictor) and beta-adrenergic (inhibitory) influences on sphincter function [11]. Also, direct hypogastric nerve stimulation results in sphincter relaxation, suggesting that the sphincter's response to sympathetic stimulation is stimulus dependent, and therefore variable [11].

As with the sympathetic innervation, the function of parasympathetic input remains uncertain, although there is some evidence for an inhibitory role [11]. Additionally, the evidence for a role of nonadrenergic, noncholinergic nerves in the relaxation of the sphincter during RAIR suggests that nerves using ATP, VIP and NO as neurotransmitters also participate in innervating the sphincter [11].

Denny-Brown once commented late in his life that he and Robertson were 'intimate' during this phase of their careers. Subjecting themselves to these highly invasive procedures attests to the dedication of these 
two young neurologists in their effort to understand the neurophysiology of defaecation and micturition. Although their paper did not indicate how their findings would be clinically beneficial, the hundreds of citations of the paper since 1935 suggest that this basic research stimulated clinical work, which has benefited patients. Unfortunately, Denny-Brown's writing style has doubtless precluded many from appreciating the ingenuity and hard work involved in developing and conducting this study. We hope our clarification here has, to some degree, rectified this situation.

\section{Acknowledgements}

We are indebted to Mrs Roberta Shadle for her work on Figs 1 and 2 .

\section{References}

1 Vilensky JA, Gilman S, Dunn E. Derek E. Denny-Brown (1901-81): His life and influence on American Neurology. J Med Biog 1998; 6: 73-8.

2 Denny-Brown D, Robertson EG. On the physiology of micturition. Brain 1933; 56: 149-90.
3 Denny-Brown D, Robertson EG. An investigation of the nervous control of defecation. Brain 1935; 58: 256-307.

4 Vilensky JA, Gilman S. Positive and negative factors in movement control: a current review of Denny-Brown's hypothesis. J Neurol Sci 1997; 151: 149-58.

5 Gilman S, Vilensky JA, Morecraft RW, Cook JA. DennyBrown's views on the pathophysiology of dystonia. J Neuro Sci 1999; 167: 142-7.

6 Vilensky JA, Robertson WM, Gilman S. Denny-Brown, Wilson's Disease and BAL (British anti-lewisite [2,3-dimercaptopropanol]). Neurology 2002; 59: 914-6.

7 Vilensky JA, Bell DR, Gilman S. 'On the physiology of micturition' by Denny-Brown and Robertson: a classic paper revisited. Urology, 2004; 64: 1-5.

8 Gowers WR. The automatic action of the sphincter ani. Proc $R$ Soc Lond 1877; 26: 77-84.

9 Guyton AC, Hall JG. (2000) Textbook of Medical Physiology. W.B. Saunders, Philadelphia.

10 Geetinder K, Gardiner A, Duthie GS. Rectoanal reflex parameters in incontinence and constipation. Dis Colon Rectum 2002; 45: 928-33.

11 Sangwan YP, Solla J. Internal anal sphincter. Advances and insights. Dis Colon Rectum 1998; 41: 1297-311.

12 Lubowski DZ, Nicholls RJ, Swash M, Jordan MI. Neural control of internal anal sphincter function. B J Surg 1987; 74: $668-70$. 